\title{
Leiomyosarcoma of the Tracheostomy Site in a Patient with History of Laryngeal Squamous Cell Carcinoma
}

\author{
Zainub Ajmal $^{a} \quad$ Abdul Moiz Khan ${ }^{a}$ Lezah McCarthy ${ }^{b}$ Allison Lupinettic \\ Syed Mehdid \\ aDepartment of Internal Medicine, Albany Medical Center, Albany, NY, USA; ${ }^{b}$ Department \\ of Pathology, Stratton Veteran Affairs Medical Center, Albany, NY, USA; 'Department of \\ Otolaryngology, Stratton Veteran Affairs Medical Center, Albany, NY, USA; dDepartment of \\ Hematology and Oncology, Stratton Veteran Affairs Medical Center, Albany, NY, USA
}

\author{
Keywords \\ Head and neck cancer · Soft tissue sarcoma - Leiomyosarcoma · Tracheal malignancy . \\ Radiation therapy · Surgery
}

\begin{abstract}
Leiomyosarcoma (LMS) of the trachea is an extremely rare malignancy with only a few reported cases in English literature. As such the diagnosis can be frequently missed or delayed. We present a case of a 69-year-old male who underwent tracheostomy for airway obstruction secondary to glottic squamous cell carcinoma and treated definitely with radiation therapy. Subsequently, the patient developed LMS of the tracheostomy site. The case further details multiple risk factors that could contribute to development of LMS including radiation exposure, prior malignancy, and chronic inflammation. These risk factors have been well established for LMS in other sites but less so in the head and neck region, which is the subject of our discussion. We also review the current guidelines for head and neck as well as limb sarcomas and discussed role of surgery or radiation and their accompanying challenges in management of this rare malignancy.
\end{abstract}

\section{Introduction}

Leiomyosarcoma (LMS) is a soft tissue sarcoma (STS) that arises from smooth muscles. It commonly occurs in gastrointestinal tract, uterus, and retroperitoneal structures and is rare in the head and neck given the paucity of smooth muscles [1]. The origin is thought to be

\section{Karger ${ }^{\prime \prime}=$}


erector pili, vascular tunica media, or from pluripotent mesenchymal cells [2]. Tracheal malignancy in itself is fairly uncommon comprising $0.1-0.4 \%$ of all malignancies, of which majority are squamous or adenoid cystic carcinomas. As such, tracheal LMS is an extremely rare entity with only a few cases reported [3, 4]. It may be a primary tumor or metastatic from thoracic or extra-thoracic sites. Youssef et al. [5] reported a case of tracheal LMS with an associated retroperitoneal mass, while others have reported an isolated finding of tracheal LMS presenting as stridor [5,6]. The tumor tends to spread hematogenously with lymphatic spread rarely reported. Common sites of metastasis from head and neck LMS are lung, liver, and bone.

While not reported for trachea, LMS at other sites in head and neck has been associated with radiation exposure, neurofibromatosis type I, immunodeficiency, prior histologically different malignancy at same site, and history of retinoblastoma [2]. Treatment is primarily surgical resection with or without postoperative radiation therapy (RT). Prognosis is generally poor and depends largely on amenability to resection and tumor grade.

We present a case of a 69-year-old patient who developed LMS at tracheostomy site 6 months after being treated definitively with RT for a glottic squamous cell carcinoma (SCC). Unfortunately, he was not a candidate for surgical resection or reirradiation and hence opted for palliative and hospice care. We will discuss clinical presentation, risk factors, and treatment modalities for this rare malignancy.

\section{Case Presentation}

A 69-year-old male presented to the otolaryngology (ENT) clinic with 6 months of progressive hoarseness without stridor or dyspnea. Flexible fiberoptic nasopharyngolaryngoscopy showed an exophytic mass involving anterior commissure and anterior one-third of both vocal cords. A neck computerized tomography (CT) scan indicated at 6-7 mm nodularity at the anterior one-third of the vocal cords without any significant lymphadenopathy. Patient subsequently did not comply with his appointments and a biopsy could not be performed. He returned to ENT clinic 6 months later with worsening hoarseness, stridor, dyspnea, and dysphagia. Flexible fiberoptic nasopharyngolaryngoscopy showed interval increase in size of the mass, now occupying $>75 \%$ of the anterior airway. Posterior airway was patent. He underwent awake local tracheostomy with direct laryngoscopy and a biopsy of the mass that showed invasive SCC with spindle cell differentiation. Immunohistochemical staining (cytokeratin AE1/AE3) for pankeratin on the specimen was strongly positive (Fig. 1). Post procedure, the patient was noted to have increased drainage from tracheostomy site and aspirates were positive for Candida albicans, subsequently treated with fluconazole. Fluorodeoxyglucose-positron emission tomography scan showed no nodal or metastatic disease and final stage was decided as stage II T2N0M0. Based on NCCN guidelines, decision was made to treat with definitive RT for a total of 7,000 cGy in doses of $200 \mathrm{cGy} /$ day. Posttreatment CT scan showed no residual tumor. Repeat tracheoscopy however showed persistent airway compromise due to paresis and edema of the true vocal cords and hence tracheostomy was not reversed.

Patient symptomatically improved over the next 2 months. Interval monthly tracheoscopies revealed patent carina, skin breakdown on chest and submental area and superficial excoriation on posterior tracheal wall due to right-sided tracheal tube deviation from significant neck flexion. However, no intraluminal or peristomal mass was seen.

Three months after completion of radiation, patient was admitted with increased purulent discharge concerning for tracheitis and was treated with a course of antibiotics. He improved clinically but continued to experience dyspnea in addition to orthopnea. This prevented him

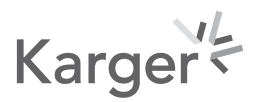




\section{Case Reports in Oncology}

\begin{tabular}{l|l}
\hline Case Rep Oncol 2021;14:912-917 \\
\hline DOI: 10.1159/000516797 & $\begin{array}{l}\text { @ 2021 The Author(s). Published by S. Karger AG, Basel } \\
\text { www.karger.com/cro }\end{array}$ \\
\hline
\end{tabular}

Ajmal et al.: Tracheal Leiomyosarcoma

Fig. 1. a $H$ and $E$ staining $(\times 200)$ shows small infiltrative islands of squamous cells. Prominent squamous atypia including pyknotic keratinocytes and nuclear atypia such as prominent nucleoli are seen. The findings are diagnostic of invasive SCC. $\mathbf{b}$ Immunohistochemical staining (cytokeratin AE1/AE3) for pankeratin shows brown chromogen staining of cells of squamous lineage. SCC, squamous cell carcinoma.

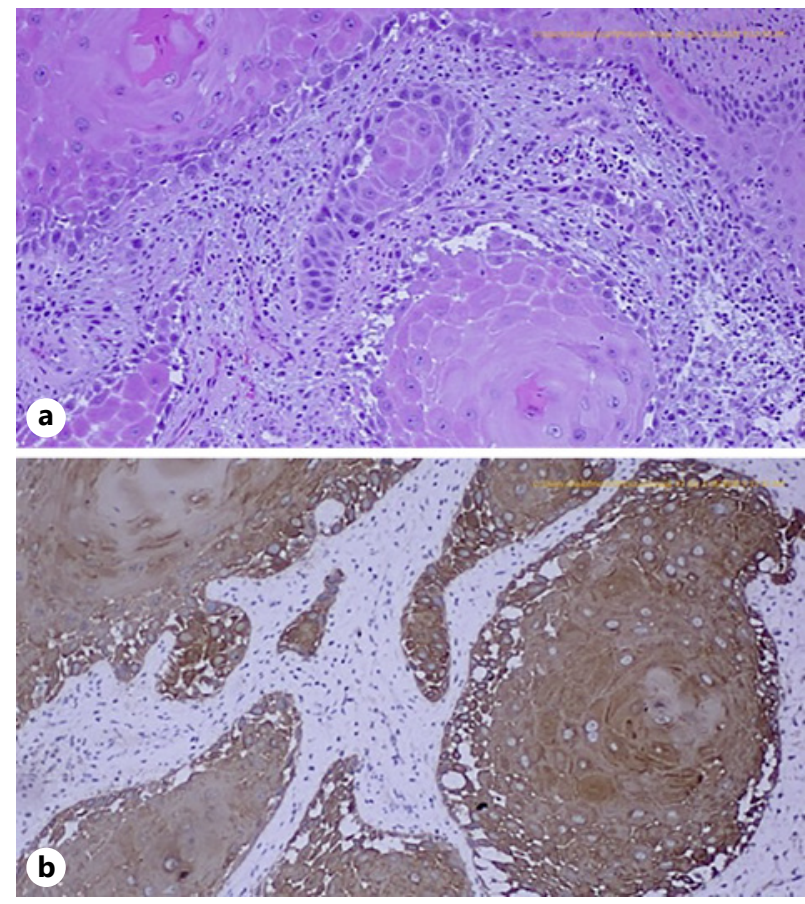

from getting a posttreatment positron emission tomography scan. Patient also had placement of gastric feeding tube given his very poor oral intake and intermittent dysphagia. Surveillance monthly tracheoscopy was notable for a superficial granuloma near the tracheal base plate but no masses.

Six months after the completion of radiation, he was readmitted with tracheitis and neck and facial swelling. Pus cultures from tracheostomy revealed methicillin-resistant Staphylococcus aureus that was treated with appropriate antibiotics. Repeat tracheoscopy revealed a peristomal mass from 12 to 6 o'clock, pushing the tracheostomy tube inferiorly and to the right. Biopsy of the mass was showed malignant spindle cell neoplasm. There were increased mitotic figures (up to 16 per $10 \mathrm{HPF}$ ) with areas of necrosis. Immunohistochemical staining was strongly positive for actin and equivocal for desmin while staining for S100, CD34, and pankeratin was negative (see Fig. 2). Together with the morphology, this was diagnostic of LMS.

Possible treatment modalities including surgical resection, radiation, and chemotherapy were considered. However, based on the size and location of the mass, the patient was not deemed to be a surgical candidate. Given his history, further RT was not an option and because of the relatively chemoresistant nature of this malignancy, systemic treatment was not chosen. After detailed discussions with the patient, family, and with palliative care team on board, patient decided to opt for community hospice care. He passed away 1 month after the diagnosis of the LMS.

\section{Discussion}

Tracheal malignancy usually presents with cough, wheezing, stridor, dyspnea, hemoptysis or acute tracheal obstruction, and airway compromise. The fact that these symptoms frequently overlap with more common respiratory pathologies including asthma or chronic bronchitis and owing to the rarity of the disease itself, the diagnosis of tracheal LMS is often delayed. For example, Balmaceda reported a case of tracheal LMS initially thought to be 


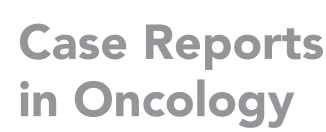

Case Reports in Oncology

\begin{tabular}{l|l}
\hline Case Rep Oncol 2021;14:912-917 \\
\hline DOI: 10.1159/000516797 & $\begin{array}{l}\text { @ 2021 The Author(s). Published by S. Karger AG, Basel } \\
\text { www.karger.com/cro }\end{array}$ \\
\hline
\end{tabular}

Ajmal et al.: Tracheal Leiomyosarcoma
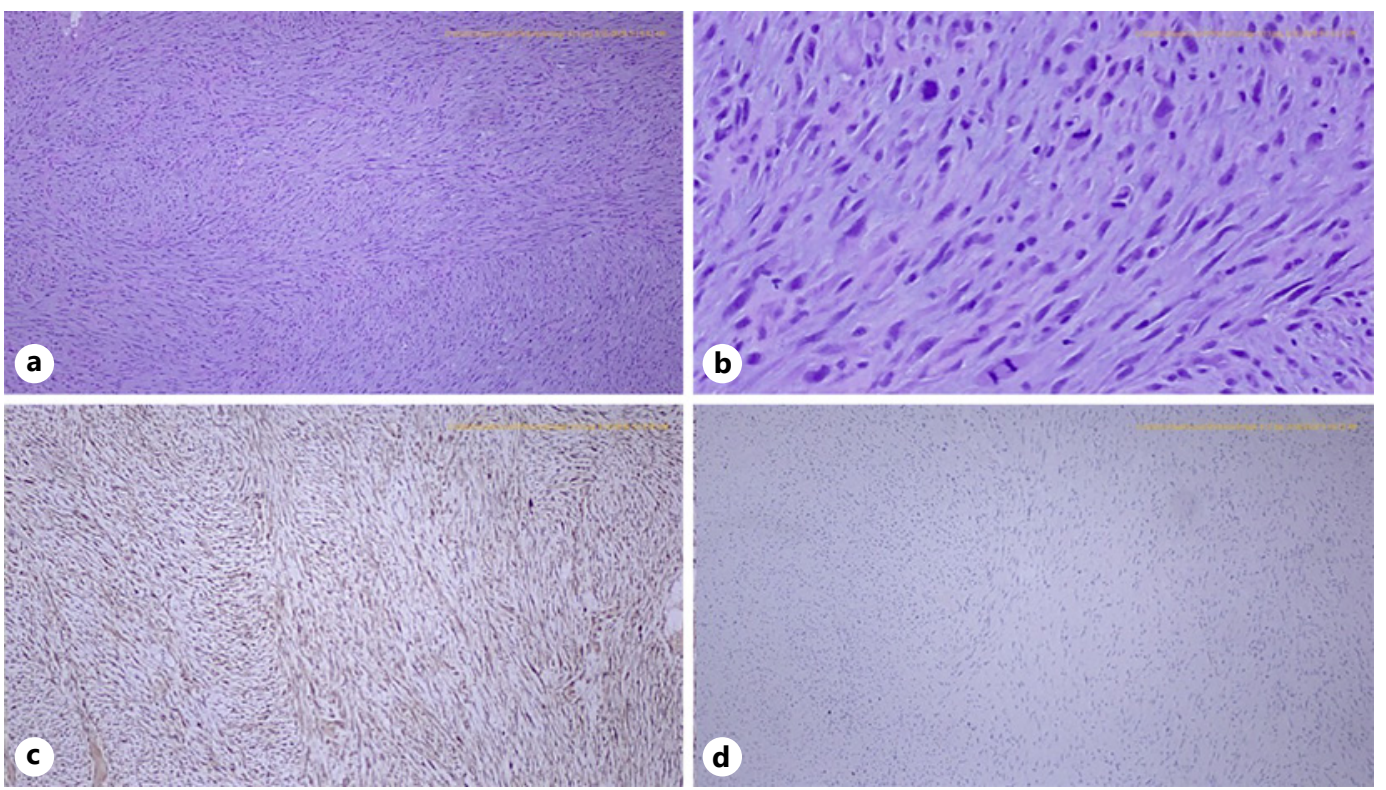

Fig. 2. a $\mathrm{H}$ and $\mathrm{E}$ staining $(\times 200)$ shows streaming fascicles of atypical spindle cells. No obvious squamous cells are present. $\mathbf{b} \mathrm{H}$ and $\mathrm{E}$ staining $(\times 400)$ shows atypical spindle cells with abundant mitotic figures. This along with the elevated mitotic figures and the atypia are consistent with sarcoma. $\mathbf{c}$ Immunohistochemical staining. The spindle cells stained positive with actin (the brown chromogen) which proves the smooth muscle lineage of the cells. This along with the overall morphology is diagnostic of LMS. $\mathbf{d}$ Immunohistochemical staining with cytokeratin AE1/AE3 (pankeratin) is negative which is inconsistent with squamous cell lineage. LMS, leiomyosarcoma.

asthma until flow volume loop indicated fixed obstruction and imaging revealed tracheal mass [7]. Appropriate diagnostic tools include CT imaging of neck with or without direct visualization with rigid bronchoscopy followed by fine-needle aspiration biopsy. In general, LMS on histology shows spindle-shaped cells with high mitotic rate (usually $>5$ per $10 \mathrm{HPF}$ ), focal necrotic regions, and varying degrees of nuclear pleomorphisms. Immunohistochemistry is an essential part of confirming the diagnosis and tumors generally stain positive for desmin, h-caldesmon, and smooth muscle actin [8].

Radiation exposure is a well-established risk factor for STS; however, it is rarely reported for LMS and to our knowledge never for tracheal LMS. Traditionally, the diagnostic criteria of radiation-induced sarcomas included histological confirmation, location within the radiation field and at least a 5-year interval between radiation and appearance of sarcoma. However, more recently, experts have suggested reducing the duration of latency to as less as 6 months [9-11]. Though our patient's diagnosis was histologically confirmed, and tumor was in the radiation field, the time of appearance of LMS was within 5 months of radiation. Therefore, it is difficult to say how much contribution radiation had in the sarcomagenesis. When present, radiation-induced STS have a worse prognosis than their counterparts partly because treatment options are frequently limited to surgery alone. The anatomic site is often not amenable to reirradiation, LMS in general is not chemosensitive and surgery with wide margins is often not feasible due to proximity to vital structures [12].

Although most LMS arise de novo, a history of prior histologically distinct malignancy is a known risk factor for LMS. There have been reports of metachronous LMS developing at site of previously treated SCC as well as synchronous LMS and SCC in larynx $[13,14]$. In our case, the LMS appeared after the successful treatment of the SCC.

\section{Karger'}


Another potential risk factor in our patient, and for sarcomagenesis in general, is chronic inflammation at the tumor site. While the most common inflammation-associated sarcomas are Kaposi sarcoma and malignant fibrous histiocytoma, inflammation in any setting causes multiple cell lines including fibroblasts and epithelial cells to proliferate through release of cytokines, chemokines, and growth factors. Failure to exit this cycle of remodeling at any point results in chronic inflammation and persistent proliferative stimuli that can cause both tumor initiation and progression. While not reported for LMS, there are case reports of developing other types of STS at sites of chronic inflammation including fibrosarcoma in setting of chronic osteomyelitis and rhabdomyosarcoma at site of chronic wound from bullet injury [15, 16]. Our patient similarly presented with chronic nonhealing ulcer at tracheal base plate and multiple incidences of tracheitis. However, the duration of inflammation leading to malignancies is much more protracted than our patient.

There is no consensus on the appropriate management for tracheal LMS; however, surgical resection with wide margins remains first-line treatment for localized disease. RT is recommended postoperatively in high-grade tumors or where negative margins cannot be achieved. It may also be used in the preoperative setting for local disease control in large tumors. Use of chemotherapy remains controversial for LMS particularly in the head and neck region. Majority studies advocating benefit of systemic treatment group STS together making it difficult to ascertain benefit in any 1 particular histologic subtype. At present, chemotherapy does not seem to have role in local or systemic disease treatment [17]. LMS has the worst prognosis among head and neck STS. Prognosis for tracheal LMS specifically is difficult to ascertain due to the limited available data. In general, it depends on ability to achieve negative surgical margins and tumor grade $[18,19]$.

\section{Conclusion}

LMS of trachea is an extremely rare and aggressive malignancy that should be considered as a differential particularly in patients with prior history of malignancy, radiation exposure, or chronic inflammation. As of now, surgical resection seems to be the only available option associated with long-term survival.

\section{Acknowledgements}

This material is the result of work supported with resources and the use of facilities at the Albany Stratton Veteran Affairs Medical Center, Albany, NY, USA. However, no financial aid/grant has been provided. It is hereby declared that in addition to the aforementioned, the authors carry no affiliations with or involvement in any organization or entity with any financial or nonfinancial interest. It is also declared that this research did not receive any grant from any funding agencies in the public, commercial, or not-forprofit sectors. The authors of this case report also did not seek help from any writing assistance services.

\section{Statement of Ethics}

Written informed consent was obtained from the patient for publication of this case report and any accompanying images. No patient identifier was used in the case description and included figure.

\section{Karger'}




\section{Conflict of Interest Statement}

It is also declared that this research did not receive any grant from any funding agencies in the public, commercial, or not-for-profit sectors.

\section{Author Contribution}

Dr. Zainub Ajmal is the primary author of this case report who contributed to every section. Dr. Abdul Moiz Khan worked on the discussions. Dr. Lezah McCarthy is the pathologist of this case who not only provided the histological images but also their interpretation. Dr. Lupinetti is the patient's primary otolaryngologist. Dr. Syed Mehdi is the primary oncologist of the patient in our case. He served as the preceptor, providing us with the necessary resources to write this case. He also reviewed and proofread all the sections.

\section{References}

1 Workman AD, Farquhar DR, Brody RM, Parasher AK, Carey RM, Purkey MT, et al. Leiomyosarcoma of the head and neck: a 17-year single institution experience and review of the National cancer data base. Head Neck. 2018;40(4):756-62.

2 Saluja TS, Iyer J, Singh SK. Leiomyosarcoma: prognostic outline of a rare head and neck malignancy. Oral Oncol. 2019;95:100-5.

3 Ahn Y, Chang H, Lim YS, Hah JH, Kwon TK, Sung MW, et al. Primary tracheal tumors: review of 37 cases. J Thorac Oncol. 2009;4(5):635-8.

4 Grillo HC, Mathisen DJ. Primary tracheal tumors: treatment and results. Ann Thorac Surg. 1990;49(1):69-77.

5 Youssef F, Godbole R, Longoria J, Gallegos N, Kim G, Davoudi M. Tracheal leiomyosarcoma causing critical airway obstruction. Chest. 2017;152(4):A657.

6 Fleetham JA, Lynn RB, Munt PW. Tracheal leiomyosarcoma: a unique cause of stridor. Am Rev Respir Dis. 1977;116(6):1109-12.

7 Balmaceda D, Ciccolo M, Ma Y, Baydur A. Leiomyosarcoma of the trachea masquerading as chronic asthma. Respiration. 2002;69(2):165.

8 Serrano C, George S. Leiomyosarcoma. Hematol Oncol Clin North Am. 2013;27(5):957-74

9 Cahan WG, Woodard HQ, Higinbotham NL, Stewart FW, Coley BL. Sarcoma arising in irradiated bone; report of 11 cases. Cancer. 1948;1(1):3-29.

10 Gladdy RA, Qin L-X, Moraco N, Edgar MA, Antonescu CR, Alektiar KM, et al. Do radiation-associated soft tissue sarcomas have the same prognosis as sporadic soft tissue sarcomas? J Clin Oncol. 2010;28(12):2064-9.

11 Demirkan F, Unal S, Cenetoğlu S, Cinel L. Radiation-induced leiomyosarcomas as second primary tumors in the head and neck region: report of 2 cases. J Oral Maxillofac Surg. 2003;61(2):259-63.

12 Giannini L, Incandela F, Fiore M, Gronchi A, Stacchiotti S, Sangalli C, et al. Radiation-induced sarcoma of the head and neck: a review of the literature. Front Oncol. 2018;8:449.

13 Kara E, Cetik MF, Tuncer U, Uguz A. A rare association of leiomyosarcoma with squamous cell carcinoma of the larynx: two cases. J Laryngol Otol. 2013;127(8):828-31.

14 Ismi O, Arpaci RB, Puturgeli T, Ozcan C, Gorur K. Leiomyosarcoma of larynx as a metachronous tumor of squamous cell carcinoma: an unusual case. J Voice. 2016;30(2):242-5.

15 Morris JM, Lucas DB. Fibrosarcoma within a sinus tract of chronic draining osteomyelitis. case report and review of literature. J Bone Joint Surg Am. 1964;46(4):853-7.

16 Bar Y, Merimsky 0. Soft-tissue sarcoma following traumatic injury: case report and review of the literature. Front Oncol. 2017;7:134.

17 Grimer R, Judson I, Peake D, Seddon B. Guidelines for the management of soft tissue sarcomas. Sarcoma. 2010; 2010:506182.

18 Eppsteiner RW, DeYoung BR, Milhem MM, Pagedar NA. Leiomyosarcoma of the head and neck: a populationbased analysis. Arch Otolaryngol Head Neck Surg. 2011;137(9):921-4.

19 Mattavelli D, Miceli R, Radaelli S, Mattavelli F, Cantù G, Barisella M, et al. Head and neck soft tissue sarcomas: prognostic factors and outcome in a series of patients treated at a single institution. Ann Oncol. 2013;24(8): 2181-9. 